\title{
Una oportunidad de mayor legitimidad de las ONG a través de la transparencia on-line Experiencia de las ONG españolas y mexicanas
}

\section{María del Carmen Caba Pérez \\ Universidad de Almería ccaba@ual.es}

María del Mar Gálvez Rodríguez. Universidad de Almería maregalvez@hotmail.com

Manuel López Godoy Universidad de Almería malopez@ual.es

\section{Resumen}

En los últimos años se ha incrementado la necesidad de una mayor transparencia que fortalezca la confianza hacia las ONG y responda a las necesidades informativas de los diferentes grupos de interés o stakeholders; para favorecer el acceso a la información se considera Internet como elemento estratégico de comunicación. Siguiendo una línea de investigación descriptiva, en este artículo se analizan la divulgación de información a los stakeholders de ONG españolas y mexicanas a través de sus web con el fin de valorar su nivel de transparencia. Las ONG españolas analizadas son aquellas que se sometieron, voluntariamente, a la verificación de su transparencia según los criterios de la Fundación Lealtad. Entre las principales conclusiones de este trabajo destaca que tanto las ONG españolas como mexicanas deben realizar mayores esfuerzos de transparencia. No obstante, existen diversos organismos públicos y privados, como el caso de la Fundación Lealtad, que están contribuyendo de manera significativa en el fomento del uso de webs como mecanismo de mejora de transparencia del sector.

Palabras clave: transparencia, divulgación, oNG, Internet 


\title{
An opportunity for greater legitimacy of NGOs through the online trans- parency: Experience of Spanish and Mexican NGOs
}

\begin{abstract}
In recent years the need of a major transparency to enhance NGOs' trust and to respond the information needs In recent years the need of a major transparency to enhance NGOs' trust and to respond the information need of their stakeholders has increased. The Internet is considered as a strategic communication tool to encourage the information access. Following a descriptive line of research, this paper aims at analyzing the level of transparency of Spanish and Mexican NGOs. Regarding this, the information disclose to their stakeholders through their web pages is analyzed. This study has been developed based on a sample of Spanish NGOs that were analyzed voluntarily by the Lealtad Foundation. As the main findings, both the Spanish NGOs and the Mexican NGOs should make a major effort to disclose information through the Internet in order to improve the degree of transparency. Despite these facts, it has to be pointed out, that there are public and private organizations, like Lealtad Foundation, that are promoting the use of web pages as a mechanisms to enhance the transparency of this sector of their stakeholders has increased. The Internet is considered as a strategic communication tool to encourage the information access.
\end{abstract}

Keywords: transparency, disclosure, Internet, NGO

\section{Introducción}

En los últimos años ha proliferado el número de ONG como instituciones de referencia ética en la sociedad (Rathgeb, 2008); este reconocimiento es además fundamental para la supervivencia de éstas, pues gran parte del capital humano y financiación de la organización depende de la ayuda desinteresada de donantes (administración pública y particulares) y voluntarios (Seaman, 2006).

De acuerdo con la legitimidad de las ONG, cabe señalar que ésta no es permanente, más bien depende de una adecuada transparencia que muestre que los objetivos de la organización no sólo benefician a unos pocos, sino que persiguen fines de carácter plural (Berman, 2006). En este sentido, los recientes casos de fraude detectados en el sector han cuestionado su labor al servicio de la sociedad (Greenlee et al.,2007); por tanto, para combatir esta falta de credibilidad, Herranz de la Casa (2007) pone de manifiesto la necesidad de un mayor acceso a la información tanto sobre aspectos de la organización como económicos, además de las actividades que realizan. 
Por otro lado, es imprescindible que la elaboración de esta información esté al alcance de la sociedad; es por ello que Internet es considerado como un elemento estratégico para la divulgación de información de las ONG (Pérez y Mateos, 2006; Ingenhoff y Koelling 2007). Sin embargo, en líneas generales, las ONG hacen un escaso uso del potencial que tienen las páginas web como transmisoras de información relevante hacia a sus stakeholders (Kang y Norton, 2004).

De acuerdo con los mecanismos de rendición de cuentas voluntarios para el fomento de la transparencia del sector, cabe señalar que las propias ONG están llevando a cabo diversas propuestas ${ }^{1}$ e incluso foros internacionales ${ }^{2}$. En el caso español señalamos, entre otras, los principios de transparencia del código de conducta de la Fundación Lealtad. Esta entidad sin ánimo de lucro no sólo ha conseguido que cada año aumente el número de ONG españolas que solicitan ser analizadas según sus recomendaciones en materia de transparencia, sino que actualmente está trabajando para adaptar su metodología de análisis a las ONG latinoamericanas. Concretamente, una de sus actuaciones más recientes es la promoción de la transparencia en México; de esta forma, dicha fundación pretende apoyar a las ONG mexicanas en el desarrollo e implantación de principios de transparencia con el fin de fomentar la confianza en el sector por parte de la sociedad mexicana y de los numerosos donantes españoles.

Con estos precedentes, este trabajo persigue realizar un análisis del grado de transparencia on-line de las de las ONG españolas y mexicanas.

\section{La transparencia y las ONG}

\section{La transparencia más que un amplio concepto a la realidad económica}

La elaboración de información no sólo es necesaria para la toma de decisiones, sino que es fundamental para responder a las necesidades informativas de los principales grupos de interés o stakeholders (donantes, gobiernos y beneficiarios) de las ONG. Fuentes (2007) señala que la información que suministran

\footnotetext{
${ }^{1}$ Ejemplos de iniciativas en Estados Unidos como Standards for Excellence elaborados por Maryland Association for Nonprofit Asssociations o Private Voluntary Organizations Standards de Interaction; en Europa con la Carta della Donazione de la Charte de deontologie, el certificado de DZI Deutsches Zentralinstitutfür soziale Fragen; y en Latinoamérica los requisitos de transparencia de ONG por la Transparencia ó el código de conducta propuesto por Chile Transparente.

${ }_{2}^{2}$ International Commitee on Fundraising Organizations (ICFO)
} 
las ONG no cumple con la demanda informativa de los stakeholders, pues no contempla adecuadamente la gestión y el cumplimiento de los objetivos de la organización. Por su parte, Burger y Owens (2010) añaden que esta asimetría de información entre las ONG y sus stakeholders debe ser corregida a través de una mayor transparencia.

Dada la importancia de la transparencia para fortalecer la confianza en el sector (Duble, 1994; MacDonald et al., 2002; McGann y Johnstone, 2006; Sanz y Koc 2006), es conveniente detenerse en analizar los diferentes aspectos que sustentan este concepto. La transparencia de las ONG es el grado de información y la actitud en que se afronta la rendición de cuentas (Grabulosa, 2007). Bonbrigth (2007) además señala que esta necesidad de información en las ONG está dirigida a los diferentes stakeholders. En este sentido, Medina (2009) pone de manifiesto que la transparencia está enfocada en diversas direcciones: a) externa, dirigida a donantes, socios y beneficiarios; b) interna, orientada hacia sus trabajadores, ya sea personal contratado como voluntariado; y c) horizontal, con el fin de reforzar las alianzas y relaciones con otras ONG y organismos oficiales.

Por su parte, Hooper et al. (2008) señalan que la transparencia de las ONG conlleva la elaboración de información de carácter financiero. Autores como Vaccaro y Madsen (2009) inciden en la transparencia como el grado de información a los diferentes stakeholders sobre las actividades que realizan, así como el fomento de una actitud más ética hacia el buen gobierno de la organización.

En consecuencia, la transparencia ha pasado de ser un tema operativo a transformarse en un aspecto estratégico, ya que se relaciona con la propia identidad de la organización, con la presencia de mecanismos para la evaluación y con la verificación de las actuaciones mediante sistemas de información y comunicación seguros (Ruiz et al., 2008).

La fortaleza de la legitimidad de la ONG depende, entre otros aspectos, de una rendición de cuentas que responda a las expectativas de los stakeholders de la organización (Lister, 2003). Para Guberty (2009), así como para Burger y Owens (2010), las ONG deben elaborar información que permita el control externo de la organización. De este modo, los stakeholders consideran los mecanismos para la rendición de cuentas como una señal para distinguir a las ONG más transparentes. 
Entre los mecanismos de rendición de cuentas que aplican las ONG, autores como O’Rourke (2006) y Argandoña (2007) señalan los códigos de conducta que son procesos de carácter voluntario que comprenden una serie de valores para la adecuada gestión interna y externa de la organización. Estos códigos de conducta contribuyen al éxito de la misión de las ONG y al incremento de la participación de los stakeholders. En definitiva, son una herramienta que garantiza una adecuada transparencia para fortalecer la credibilidad de los stakeholders hacia las ONG.

Por otro lado, las organizaciones revelan voluntariamente información para demostrar su compromiso social ante la comunidad y así legitimarse (Campbell, 2006). En este sentido, autores como Naudé et al. (2004), Ingenhoff y Koelling (2007), y Ozcelik (2008) ponen de manifiesto la utilidad de las nuevas tecnologías, y en especial Internet, para la difusión de la información.

La web es un instrumento que ofrece una gran ventaja competitiva a las ONG porque gracias a su disponibilidad, bajo coste y accesibilidad favorece que los donantes y voluntarios decidan su implicación en función de la calidad y cantidad de la información suministrada en la red (Hackler, 2007; Lee et al., 2001; Naudé et al 2004). Autores como Wanderley et al. (2008) añaden que el uso de Internet permite a las ONG ofrecer información actualizada e interactuar con sus usuarios, de manera que se responda mejor a las necesidades informativas de los diferentes stakeholders.

De acuerdo con el contenido de los portales web, Peregrine y Schwartz (2004) ponen de manifiesto que las páginas web son un medio perfecto para mostrar el comportamiento ético de las ONG, por lo que recomiendan el uso de esta herramienta para la divulgación de información organizacional, financiera e información que muestre las políticas para evitar el conflicto de intereses.

\section{La transparencia y la realidad de las ONG españolas y mexicanas}

En España es indudable la presencia de las ONG como uno de los actores sociales más relevantes del país; sin embargo, estudios sobre el sector en ese país ponen de manifiesto la dificultad en la recopilación de datos para determinar en términos cuantitativos tanto su peso económico como el número de organizaciones que pertenecen a este colectivo (García, 2004; Ruíz, 2001). Según Ruiz (2001), en España se encuentran alrededor de 253.507 organizaciones destinadas a fines no lucrativos 
y, con base en el estudio internacional realizado por Salomon et al. (2001), representan un 5.2\% del PIB español.

Ruíz (2001) señala que para conocer el desarrollo de las ONG en España es fundamental considerar el contexto histórico, social, económico y legal en el que se desenvuelven estas organizaciones. Su estudio muestra que la sociedad española se caracteriza por su hegemonía social, el catolicismo, el corporatismo, la democratización, la memoria histórica y la laización/ racionalización del mundo. En el aspecto económico señala que la mayor fuente de ingresos procede de las cuotas y pagos de servicios y del apoyo del sector público.

Por otro lado, en México existen cerca de 20.000 entidades destinadas a fines sociales $^{3}$ y, según el estudio realizado por Salomon et al. (2001), representan el 0.5\% del PIB mexicano. Entre sus ámbitos de actuación, Treviño (2004) destaca su labor de defensa ante los derechos humanos y la democracia, pues México es uno de los países con mayores índices de corrupción. ${ }^{4}$ Para la realización de sus proyectos, Sorj (2007) señala las dificultades económicas a las que se enfrentan las ONG autóctonas, por lo que es de gran importancia la cooperación internacional de ONG y otras instituciones procedentes, en su mayoría, de Estados Unidos y Europa. Brumley (2010) añade que las peculiaridades políticas y la dificultad de sus relaciones con los gobiernos del país han provocado que el desarrollo del sector sea más lento en comparación con otros países.

A pesar de su contribución a los cambios sociales, se han detectado tanto en México (Neal, 2008) como en España (Medina, 2009) casos de irregularidades en la gestión de las ONG, lo que ha cuestionado su independencia y legitimidad como entidades al servicio del bienestar social. Por tanto, una mayor transparencia no solo fomentará las buenas prácticas en la gestión de estas organizaciones sino que mejorará su imagen ante los diferentes stakeholders.

De acuerdo con los mecanismos de rendición de cuentas para garantizar una adecuada transparencia, hay que señalar que en España las ONG están sujetas a normativas que principalmente se centran en la elaboración de información de ca-

\footnotetext{
${ }^{3}$ Según datos facilitados por la Fundación Lealtad http://www.fundacionlealtad.org/web/jsp/medios/detalleNotic ia?idNoticia $=1295 \&$ idTipoNoticiaSelec $=2$

${ }^{4}$ Informe de Transparencia Internacional , 2008
} 
rácter financiero, ${ }^{5}$ como el balance de situación y cuenta de pérdidas y ganancias. No obstante, las propias ONG están llevando a cabo propuestas para fomentar la elaboración de aspectos económicos, las actividades que realizan y aspectos organizativos (Marcuello et al., 2007; Moneva y Bellostas, 2007).

Entre las iniciativas puestas en marcha se encuentra la Guía de Transparencia y Buenas Prácticas elaborada por la Fundación Lealtad, entidad sin ánimo de lucro creada en 2001, cuyo principal objetivo es el fomento de la credibilidad de la sociedad hacia las ONG. Además, esta entidad es miembro de la junta directiva de International Commitee on Fundraising Organizations (ICFO), foro internacional para la discusión y el debate sobre los requisitos y criterios para el análisis de las ONG.

La fundación analiza el grado de transparencia de las ONG a través del cumplimiento de nueve de principios que abordan aspectos de transparencia organizativa, de las actividades que realizan, voluntariado, grado de visibilidad y aspectos económicos referentes a la transparencia en las fuentes de financiación como a la presentación de las cuentas anuales y cumplimiento de las obligaciones legales (figura 1). Esta evaluación es voluntaria; por tanto, se realiza sobre aquellas ONG que, por iniciativa propia, entregan la información solicitada. Tras la evaluación se remite un certificado e informe de transparencia que está disponible en la página web de la Fundación. Esta metodología de análisis ha tenido una gran acogida por el sector tal y como lo demuestra la evolución de ONG adheridas, de 30 en la primera guía de 2002, hasta las 139 en su última publicación de 2010.

La Fundación Lealtad ha puesto en marcha un proyecto para la adaptación de los principios de transparencia de la entidad a la realidad de las ONG en México ${ }^{6}$. De este modo, se pretende por un lado, impulsar el acceso a la información para incrementar la confianza de la sociedad mexicana en las ONG del país y, por otro lado, mejorar el seguimiento de los fondos aportados por los donantes españolas en ONG mexicanas.

\footnotetext{
${ }^{5}$ En el caso español, Real Decreto 776/1998 sobre las normas de adaptación del Plan General de Contabilidad y formación presupuestaria; Ley 50/2002 de Fundaciones; Ley 1/2002 de Asociaciones; Ley 38/2003 General de Subvenciones.

${ }^{6} \mathrm{http} / / / \mathrm{www}$. fundacionlealtad.org/web/jsp/medios/detalleNotaImp?idNota=1296\&fechaSeleccionada=
} 
Figura 1

Guía para la transparencia de las ONG

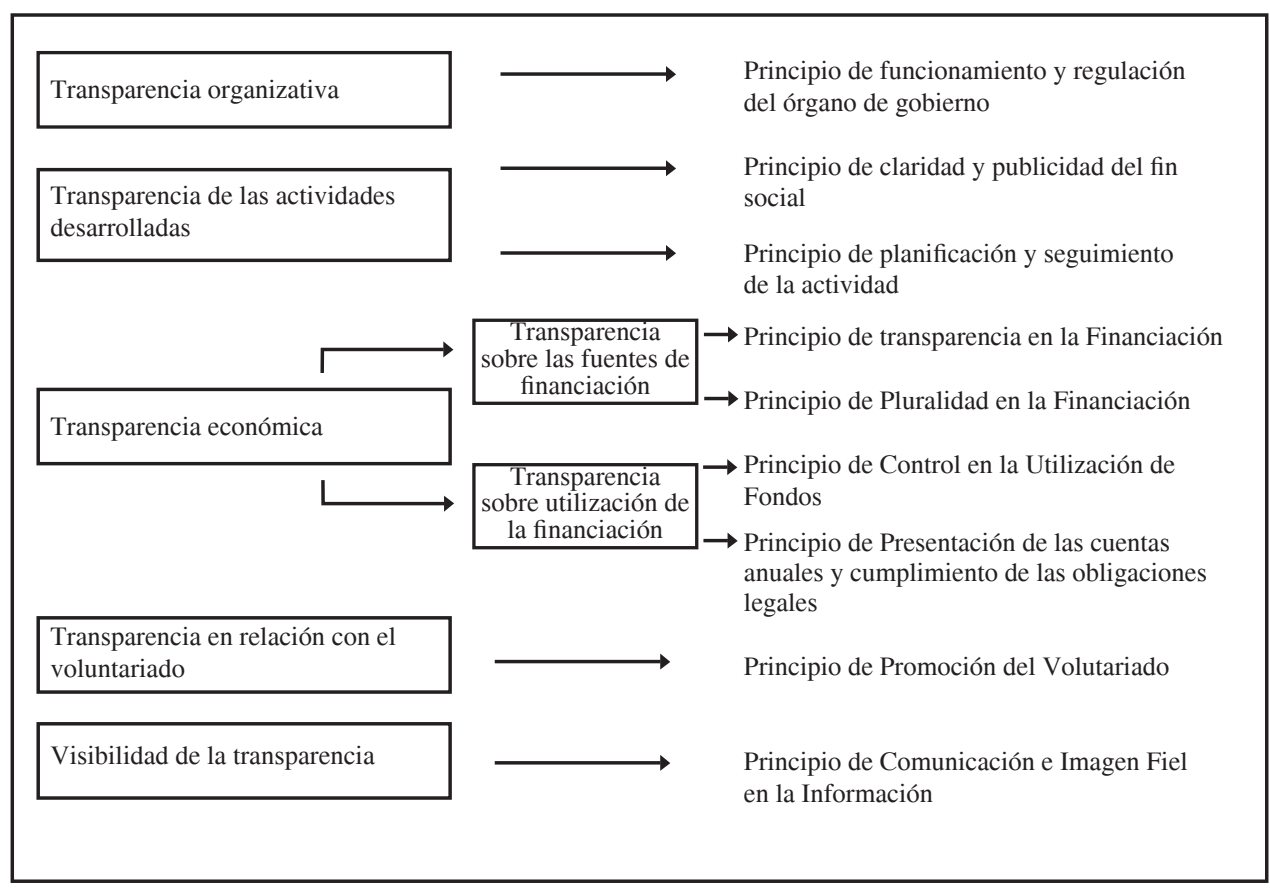

Fuente: Elaboración propia a partir de la Guía de transparencia y buenas prácticas. Fundación lealtad, 2010.

En México, una de las acciones realizadas para fomentar la transparencia ocurrió en 2002 cuando se publicó la Ley Federal de Transparencia y Acceso a la Información Pública Gubernamental ${ }^{7}$ donde, entre los requisitos de elaboración de información, se impone la divulgación mediante Internet de aspectos tales como la estructura organizacional, los objetivos, las retribuciones salariales, el presupuesto, los informes auditados y el cumplimiento del marco regulatorio. Esta normativa no incluye a las ONG como ocurre en otros países ${ }^{8}$, por lo que las ONG autóctonas como México Abierto, el Centro Mexicano para la Filantropía (CEMEFI), y algunos organismos públicos como la Universidad Iberoamericana ponen de manifiesto su preocupación por la necesidad de mecanismos de rendición de cuentas.

${ }^{7}$ Ley Federal de Transparencia y Acceso a la Información Pública Gubernamental, 11 de junio de 2002.

${ }^{8}$ Guatelama: Decreto Número 57-2008 sobre la Ley de Acceso a la Información Pública donde incluye a las ONG por ser organismos gestores de fondos públicos. 
Con base en estos precedentes, consideramos conveniente conocer la situación actual de transparencia on-line de las ONG mexicanas y españolas.

\section{Metodología}

\section{Descripción de la metodología}

Siguiendo una línea de investigación descriptiva y en línea con los trabajos previos referentes a la revelación de información corporativa (Caba y Castillo, 2002; Gandía, 2000; Larran y Giner, 2001;) y del sector público (Herawaty y Hoque , 2007; Serrano et al. 2008), se ha elaborado un Índice de Visibilidad de Transparencia en la Web (IVT).

De acuerdo con los principios de la Fundación Lealtad y la revisión de la literatura citada en el párrafo anterior, se han recogido una serie de ítems que se identifican con el concepto global de transparencia. En este sentido, tales factores quedan clasificados con aspectos sobre el ámbito organizacional, de las actividades que realizan y económico que se divide a su vez en aquellos factores que hacen referencia a la transparencia en el origen y utilización de la financiación recibida. En consecuencia, este índice de divulgación comprende, a su vez, tres índices parciales que se identifican con los tres aspectos comentados (cuadro 1).

En total, el número de ítems observados suman 29 (vt $\mathrm{v}_{\mathrm{i}}$ ), de los cuales cuatro corresponden al aspecto sobre la transparencia organizativa $\left(v t_{O}\right)$, ocho a la transparencia de las actividades $\left(v t_{A}\right)$ y diecisiete a la transparencia económica $\left(v t_{E}\right)$. Dentro de este último bloque, seis pertenecen a la transparencia en la financiación y once a la transparencia en la utilización de la financiación.

Dada la existencia de tres índices parciales $I V_{t}$, lo primero que haremos es calcular cada uno de ellos; por tanto, se computará el índice narcial organizativo $I V_{O}$, el índice parcial de transparencia de las actividades $I V_{A}$ y el índice parcial de transparencia económica $I V_{E}$. Dicho cálculo será el resultado del cociente entre la sumatoria del número total de ítems del $\left(\mathrm{vt}_{m}\right)$ que aparece en la web por el total de ítems que debiera divulgarse en cada índice parcial $\left(\mathrm{m}_{\mathrm{i}}\right)$. Posteriormente, para obtener los datos en términos relativos, multiplicaremos el importe resultante por 100 . 


$$
I V_{m}=\frac{\sum_{i=1}^{m} v t_{m}}{m_{i}} \times 100
$$

La no disponibilidad de evidencias empíricas sobre la importancia de los distintos ítems y subíndices que comprenden el Índice de Visibilidad de Transparencia en la Web (IVT) propuesto nos ha llevado a asignar a cada uno de ellos idéntico peso, aunque en la literatura existe un debate sobre la influencia de la ponderación de los índices de transparencia en los resultados de la investigación. En esta línea, Rodríguez (2004) opta por la no ponderación siguiendo, por un lado, los trabajos de Dhaliwal (1980) que señalan que toda ponderación conlleva cierta subjetividad y, por otro lado, la existencia de estudios de autores como Choi (1973), Robbins y Austin (1986), Chow y Wong-Boren (1987) que muestran similares resultados utilizando índices ponderados o no. Por tanto, los ítems que forman los subíndices del índice (IVT) se valorarán por una calificación dicotómica, de forma que se asignará un "1" a aquel ítem (irsw) que sí dispone de la información en su página Web y "0" en caso contrario. No obstante, hay que señalar que hay factores que se desglosan en diversos subítems, en este caso, y tal y como se aprecia en el cuadro 1, la puntuación de cada uno estos subítems quedará distribuida de manera equitativa de tal forma que la suma de todos ellos dará un valor máximo de " 1 ".

Así pues, el índice de transparencia en Internet quedaría determinado por:

$$
I V T=I V_{o}+I V_{A}+I V_{E}=\left(\frac{\sum_{i=1}^{4} v t_{O}}{4}+\frac{\sum_{i=1}^{8} v t_{A}}{8}+\frac{\sum_{i=1}^{17} v t_{E}}{17}\right) \times \frac{100}{3}
$$




\section{Cuadro 1}

\section{Factores analizados para análisis transparencia on-line}

\begin{tabular}{|c|c|c|}
\hline & Val. Max & Val. Mín. \\
\hline 1. Sub-ítem transparencia organizativa & 4 & 0 \\
\hline a. Se ofrece en la web información sobre: & 1 & 0 \\
\hline a.1. Número de reuniones anuales de los órganos de gobierno & 0,33 & 0 \\
\hline a.2. Temas por tratar en las reuniones anuales de los órganos de gobierno & 0,33 & 0 \\
\hline a.3. Acuerdos adoptados de los órganos de gobierno & 0,33 & 0 \\
\hline b. Respecto a los miembros del órgano de gobierno: & 1 & 0 \\
\hline b.1. ¿Son públicos los nombres de los órganos de gobierno o de dirección? & 0,2 & 0 \\
\hline b.2. ¿Son públicos las profesiones o cargos públicos de los órganos de gobierno? & 0,2 & 0 \\
\hline $\begin{array}{l}\text { b.3. ¿Son públicos la relación de parentesco y afinidad con otros miembros del órgano } \\
\text { de gobierno y con el equipo directivo de la organización? }\end{array}$ & 0,2 & 0 \\
\hline b.4. ¿Son públicos los currículos vitae de los miembros del equipo directivo? & 0,2 & 0 \\
\hline $\begin{array}{l}\text { b.5. ¿Son públicos la relación entre los miembros del órgano de gobierno } \\
\text { y los proveedores y coorganizadores de la actividad? }\end{array}$ & 0,2 & 0 \\
\hline c. ¿Son públicos en la web la renovación de los miembros del órgano de gobierno? & 1 & 0 \\
\hline $\begin{array}{l}\text { d. ¿Son públicos en la web los mecanismos aprobados por el órgano de gobierno que } \\
\text { eviten situaciones de conflicto de interés en el seno del órgano de gobierno? }\end{array}$ & 1 & 0 \\
\hline 2. Sub-ítem Transparencia sobre actividades & 8 & 0 \\
\hline a. ¿Es público en la web el fin social de la ONG? & 1 & 0 \\
\hline $\begin{array}{l}\text { b. ¿La información ofrecida sobre el fin social identifica claramente } \\
\text { y orienta sobre el campo de actividad y público a que se dirige? }\end{array}$ & 1 & 0 \\
\hline c. ¿Es público en la web el plan estratégico? & 1 & 0 \\
\hline d. ¿Es público en la web el plan anual? & 1 & 0 \\
\hline $\begin{array}{l}\text { e. ¿Son públicos en la web los informes de seguimiento y finales justificativos } \\
\text { de los proyectos desarrollados? }\end{array}$ & 1 & 0 \\
\hline $\begin{array}{l}\text { f. ¿Son públicos en la web los criterios y procesos de selección de proyectos } \\
\text { y contrapartes aprobados por el órgano de gobierno? }\end{array}$ & 1 & 0 \\
\hline g. ¿Es público en la web la memoria anual de actividades? & 1 & 0 \\
\hline $\begin{array}{l}\text { h. ¿Existe en la página web información sobre las actividades que están abiertas } \\
\text { por ser desarrolladas por voluntarios? }\end{array}$ & 1 & 0 \\
\hline 3. Sub-ítem Transparencia económica & 17 & 0 \\
\hline 3.1. Transparencia en la financiación & 6 & 0 \\
\hline a. ¿Es público en la web el coste anual de captación de fondos privados y públicos? & 1 & 0 \\
\hline b. ¿Es público en la web la recaudación anual (donaciones, socios y otras colaboraciones)? & 1 & 0 \\
\hline $\begin{array}{l}\text { c. ¿Son públicos en la web los detalles de los fondos recaudados a los principales financiadores, } \\
\text { tanto públicos como privados, y las cantidades aportadas por los mismos? }\end{array}$ & 1 & 0 \\
\hline d. ¿Son públicas en la web las imputaciones de los fondos recaudados a las actividades de cada año? & 1 & 0 \\
\hline $\begin{array}{l}\text { e. ¿En la página web se informa sobre la protección de datos } \\
\text { de los archivos de socios y donantes particulares? }\end{array}$ & 1 & 0 \\
\hline f. ¿Es público en la web las cláusulas de cesión del logotipo de la entidad a empresas e instituciones? & 1 & 0 \\
\hline 3.2. Transparencia en la utilización de la financiación & 11 & 0 \\
\hline $\begin{array}{l}\text { a. ¿Es público en la web la distribución de los gastos de funcionamiento agrupados en las } \\
\text { categorías de Captación de fondos, Programas-Actividad y Gestión-Administración? }\end{array}$ & 1 & 0 \\
\hline $\begin{array}{l}\text { b. ¿Es público en la web el destino de los fondos desglosado por cada proyecto } \\
\text { y línea de actividad de la organización? }\end{array}$ & 1 & 0 \\
\hline c. ¿Es público en la web los principales proveedores? & 1 & 0 \\
\hline d. ¿Es público en la web los coorganizadores de la actividad? & 1 & 0 \\
\hline e. ¿Es público en la web la política de Aprobación de Gastos? & 1 & 0 \\
\hline f. ¿Es público en la web el criterio de selección de proveedores aprobados por el órgano de gobierno? & 1 & 0 \\
\hline $\begin{array}{l}\text { g. ¿Es público en la web el presupuesto anual para el año siguiente } \\
\text { con la correspondiente memoria explicativa? }\end{array}$ & 1 & 0 \\
\hline h. ¿Es público en la web la liquidación del presupuesto de ingresos y gastos del año anterior? & 1 & 0 \\
\hline $\begin{array}{l}\text { i. ¿Hay información en la página web sobre el sistema de seguimiento de la voluntad } \\
\text { de los donantes sobre los fondos dirigidos? }\end{array}$ & 1 & 0 \\
\hline j. ¿Es público en la web el balance de situación y la cuenta de resultados? & 1 & 0 \\
\hline k. ¿Es público en la web el informe de auditoría? & 1 & 0 \\
\hline
\end{tabular}


En línea con Rodríguez (2004), para la comparación de la información divulgada en ambos países se ha realizado un análisis de la varianza con el test $t$ de Student que verificará si existen diferencias significativas entre los ítems que componen cada uno de los índices parciales.

\section{Ámbito de estudio}

Para la obtención de la información necesaria para determinar el grado de visibilidad de transparencia de las ONG españolas analizadas por la Fundación Lealtad $\left(\mathrm{ONG}_{E A F L}\right.$, en adelante) se ha tomado las páginas web de $130 \mathrm{ONG}$, que en 2008 decidieron someterse a la evaluación del cumplimiento de los principios de transparencia emitidos por la citada Fundación. Del total de las páginas web revisadas, entre mayo y junio de 2009, se han desestimado dos de ellas debido a los problemas de acceso que presentaban; por tanto, la muestra ha quedado finalmente mermada en $128 \mathrm{ONG}_{E A F L}$

Con respecto al nivel de transparencia on-line de las ONG mexicanas, la muestra se ha tomado del directorio de ONG mexicanas que se incluía en la página web de World Association of Non-Governmental Organizations, WANGO, y ONGinfo. Entre ambos, la muestra alcanzaba un total de 158 ONG mexicanas: 101 ONG pertenecientes a WANGO y 57 ONG de ONGinfo. Del total de cada una de las bases analizadas, entre mayo y junio de 2009, cabe destacar que 60 ONG carecían de portal web y en 18 la página no estaba disponible o se encontraba en construcción. De tal manera que la muestra total ha quedado mermada en 80 ONG mexicanas.

\section{Análisis de los resultados}

En términos globales, las ONG de ambos países disponen de escasa información en sus páginas web. No obstante, se observa que las $\mathrm{ONG}_{E A F L}$ muestran un mejor nivel de transparencia on-line $(30,28 \%)$ que las ONG mexicanas $(12,49 \%)$.

La información más suministrada en los portales Web de las $\mathrm{ONG}_{E A F L}$ y las ONG mexicanas es la concerniente a las actividades que realizan. Con respecto al resto de información analizada, las $\mathrm{ONG}_{E A F L}$ divulgan en sus páginas web mayor información de índole económica mientras que las ONG mexicanas muestran una mayor preferencia por la difusión de información organizacional (cuadro 2). 


\section{Cuadro 2}

Resultados generales transparencia

\begin{tabular}{|c|c|c|}
\hline Concepto & $\mathrm{ONG}_{E A F L}(\%)$ & $\begin{array}{c}\text { ONG } \\
\text { México }(\%)\end{array}$ \\
\hline 1.Sub-ítem Transparencia organizativa & 14,33 & 6,36 \\
\hline 2.Sub-ítem Transparencia sobre actividades & 52,73 & 28,05 \\
\hline 3.Sub-ítem Transparencia económica & 23,78 & 3,05 \\
\hline Total Transparencia & 30,28 & 12,49 \\
\hline
\end{tabular}

En relación con las diferencias significativas obtenidas entre los ítems que componen cada uno de los índices parciales, es necesario señalar que para el aspecto de la transparencia on-line de las actividades tanto las ONG mexicanas como las españolas $\left(\mathrm{ONG}_{E A F L}\right)$ tienden a revelar información sobre el fin social, sus áreas de trabajo y beneficiarios. Con respecto al resto de ítems, se comprueba que las ONG ${ }_{E A F L}$ divulgan con mayor frecuencia la memoria anual, los informes de seguimiento e información relativa a las actividades del voluntariado de la organización.

Por el contrario, es escasa la preocupación de las ONG analizadas por divulgar la implantación de procesos que justifiquen la selección de los proyectos, concretamente los resultados alcanzan un valor medio de 7,36\% para las $\mathrm{ONG}_{E A F L}$ siendo prácticamente nulo para el caso de las ONG mexicanas (cuadro 3).

\section{Cuadro 3}

\section{Resultados transparencia de las actividades que realizan}

\begin{tabular}{|c|c|c|}
\hline Concepto & $\mathrm{ONG}_{\text {EAFL }}(\%)$ & $\begin{array}{c}\text { ONG } \\
\text { México }(\%)\end{array}$ \\
\hline 2. Sub-ítem Transparencia sobre actividades & 52,73 & 28,05 \\
\hline a. ¿Es público en la web el fin social de la ONG? & $98,45 * * *$ & $87,65 * * *$ \\
\hline $\begin{array}{l}\text { b. ¿La información ofrecida sobre el fin social identifica claramente } \\
\text { y orienta sobre el campo de actividad y público a que se dirige? }\end{array}$ & $98,45 * * *$ & $74,39 * * *$ \\
\hline c. ¿Es público en la web el plan estratégico? & $20,16 * * *$ & $2,47 * * *$ \\
\hline d. ¿Es público en la web el plan anual? & 10,08 & 15,85 \\
\hline $\begin{array}{l}\text { e. ¿Son públicos en la web los informes de seguimiento y finales } \\
\text { justificativos de los proyectos desarrollados? }\end{array}$ & $61,24 * * *$ & $3,66^{* * * *}$ \\
\hline $\begin{array}{l}\text { f. ¿Son públicos en la web los criterios y procesos de selección } \\
\text { de proyectos y contrapartes aprobados por el órgano de gobierno? }\end{array}$ & $7,36^{* *}$ & $0,00 * *$ \\
\hline g. ¿Es público en la web la memoria anual de actividades? & $62,02 * * *$ & $20,99 * * *$ \\
\hline $\begin{array}{l}\text { h. ¿Existe en la página web información sobre las actividades que están } \\
\text { abiertas a ser desarrolladas por voluntarios? }\end{array}$ & $60,85 * * *$ & $21,25 * * *$ \\
\hline $\mathrm{p}<0,001^{* * *} ; \mathrm{p}<0,05^{* *} ; \mathrm{p}<0.1^{*}$ & & \\
\hline
\end{tabular}


Los resultados del índice parcial de transparencia económica de las ONG de Epaña $\left(\mathrm{ONG}_{E A F L}\right)$ y México son bajos. Sin embargo, en sendos casos coincide una mayor transparencia on-line sobre el origen de la financiación que en la utilización de la financiación.

Con respecto a las diferencias significativas de la información que analiza la transparencia del origen de la financiación, se observa que, frente a las ONG mexicanas, las $\mathrm{ONG}_{E A F L}$ son más responsables en la rendición de cuentas relativa a la recaudación anual de sus fondos, seguido de la imputación de los fondos a las actividades y notificación de sus principales financiadores. Asimismo, los datos revelan que las $\mathrm{ONG}_{E A F L}$ son reticentes en mostrar información sobre el coste de anual de la captación de los fondos, la cual es una información ausente en las páginas webs de las ONG mexicanas analizadas.

En cuanto a la utilización de la financiación, existen diversas diferencias significativas. Concretamente, las $\mathrm{ONG}_{E A F L}$ se preocupan más que las ONG mexicanas en mostrar en sus páginas webs el apoyo de los coorganizadores. Además, las ONG EAFL Son más conscientes de la necesidad de dar a conocer el estado financiero de la organización que las ONG mexicanas.

Por otro lado, se comprueba que las ONG de ambos países no muestran interés por comunicar en la web la veracidad de los registros contables, así como la ejecución de los presupuestos, por lo que es limitada la disponibilidad de esta información en las webs de las $\mathrm{ONG}_{E A F L}$ y prácticamente inexistente para las ONG mexicanas (cuadro 4). 


\section{Cuadro 4 \\ Resultados transparencia económica}

\begin{tabular}{|c|c|c|}
\hline Concepto & $\mathrm{ONG}_{E A F L}(\%)$ & $\begin{array}{c}\text { ONG } \\
\text { México }(\%)\end{array}$ \\
\hline 3. Sub-ítem transparencia económica & 23,78 & 3,05 \\
\hline 3.1. Transparencia en la financiación & 29,95 & 3,66 \\
\hline $\begin{array}{l}\text { a. ¿Es público en la web el coste anual de captación } \\
\text { de fondos privados y públicos? }\end{array}$ & $17,05 * * *$ & $0,00 * * *$ \\
\hline $\begin{array}{l}\text { b. ¿Es público en la web la recaudación anual } \\
\text { (donaciones, socios y otras colaboraciones)? }\end{array}$ & $55,81 * * *$ & $9,64 * * *$ \\
\hline $\begin{array}{l}\text { c. ¿Son públicos en la web los detalles de los fondos } \\
\text { recaudados a los principales financiadores, tanto públicos } \\
\text { como privados, y las cantidades aportadas por los mismos? }\end{array}$ & $33,33 * * *$ & $7,23 * * *$ \\
\hline $\begin{array}{ll}\text { d. ¿Son públicas en la web las imputaciones } \\
\text { de los fondos recaudados a las actividades de cada año? }\end{array}$ & $35,66 * * *$ & $12,05^{* * *}$ \\
\hline $\begin{array}{l}\text { e. ¿En la página web se informa sobre la protección de datos } \\
\text { de los archivos de socios y donantes particulares? }\end{array}$ & $34,88 * * *$ & $7,23 * * *$ \\
\hline $\begin{array}{l}\text { f. ¿Es público en la web las cláusulas de cesión del logotipo } \\
\text { de la entidad a empresas e instituciones? }\end{array}$ & 1,55 & 7,23 \\
\hline 3.2. Transparencia en la utilización de la financiación & 20,43 & 2,72 \\
\hline $\begin{array}{l}\text { a. ¿Es público en la web la distribución de los gastos } \\
\text { de funcionamiento agrupados en las categorías de Captación } \\
\text { de Fondos, Programas-Actividad y Gestión-Administración? }\end{array}$ & 9,30 & 0,00 \\
\hline $\begin{array}{l}\text { b. ¿Es público en la web el destino de los fondos desglosado } \\
\text { por cada proyecto y línea de actividad de la organización? }\end{array}$ & $27,91 * * *$ & $1,22 * * *$ \\
\hline c. ¿Es público en la web los principales proveedores? & 10,08 & 7,41 \\
\hline d. ¿Es público en la web los coorganizadores de la actividad? & $64,34 * * *$ & $7,41 * * *$ \\
\hline e. ¿Es público en la web la política de Aprobación de Gastos? & 1,55 & 0,00 \\
\hline $\begin{array}{l}\text { f. ¿Es público en la web el criterios de selección de } \\
\text { proveedores aprobados por el órgano de gobierno? }\end{array}$ & 3,10 & 1,23 \\
\hline $\begin{array}{l}\text { g. ¿Es público en la web el presupuesto anual para el año } \\
\text { siguiente con la correspondiente memoria explicativa? }\end{array}$ & 1,55 & 0,00 \\
\hline $\begin{array}{l}\text { h. ¿Es público en la web la liquidación del presupuesto } \\
\text { de ingresos y gastos del año anterior? }\end{array}$ & $17,83 * *$ & $6,10 * *$ \\
\hline $\begin{array}{l}\text { i. ¿Hay información en la página web sobre el sistema de } \\
\text { seguimiento de la voluntad de los donantes sobre los fondos } \\
\text { dirigidos? }\end{array}$ & 3,10 & 0,00 \\
\hline $\begin{array}{l}\text { j. ¿Es público en la web el balance de situación y la cuenta } \\
\text { de resultados? }\end{array}$ & $50,00 * * *$ & $6,71 * * *$ \\
\hline k. ¿Es público en la web el informe de auditoría? & $34,11 * * *$ & 0,00 *** \\
\hline $\mathrm{p}<0,001^{* * *} ; \mathrm{p}<0,05^{* *} ; \mathrm{p}<0.1 *$ & & \\
\hline
\end{tabular}

En la transparencia on-line organizativa se comprueba que las ONG analizadas de ambos países son proclives a divulgar en la red las personas que componen el órgano de gobierno. Este comportamiento se acentúa más en las $\mathrm{ONG}_{E A F L}$ que en las ONG mexicanas.

Entre la información menos disponible de este aspecto es significativa la exigua disponibilidad de información relativa a los reuniones del órgano de gobierno. En este sentido, se observa que las ONG mexicanas se preocupan más que las ONG ${ }_{E A F L}$ en publicar en sus webs los asuntos que se debaten en dichas reuniones, mientras que las $\mathrm{ONG}_{E A F L}$ muestran un mayor interés en notificar en la web el número 
de reuniones convocadas, los acuerdos pactados, así como las renovaciones de los miembros.

Finalmente, cabe destacar que es significativo el difícil acceso en la webs de información relativa a las relaciones existentes entre los miembros de gobierno y otros grupos de interés. En este aspecto, los resultados revelan que es prácticamente nula la divulgación de esta información por parte de las ONG de México y limitada para las $\mathrm{ONG}_{E A F L}$ (cuadro 5).

\section{Cuadro 5}

\section{Resultados transparencia organizativa}

\begin{tabular}{|c|c|c|}
\hline Concepto & $\mathrm{ONG}_{E A F L}(\%)$ & $\begin{array}{c}\text { ONG } \\
\text { México }(\%)\end{array}$ \\
\hline 1. Sub-ítem Transparencia organizativa & 14,33 & 6,36 \\
\hline \multicolumn{3}{|l|}{ a. Se ofrece en la web información sobre: } \\
\hline a.1. Número de reuniones anuales de los órganos de gobierno & $20,06 *$ & $11,09^{*}$ \\
\hline a.2. Temas por tratar en las reuniones anuales de los órganos de gobierno & $3,10^{*}$ & $8,62 *$ \\
\hline a.3. Acuerdos adoptados de los órganos de gobierno & $7,75^{* *}$ & $1,23 * *$ \\
\hline \multicolumn{3}{|l|}{ b. Respecto a los miembros del órgano de gobierno: } \\
\hline b.1. ¿Son públicos los nombres de los órganos de gobierno o de dirección? & $75,97 * * *$ & $53,66 * * *$ \\
\hline b.2. ¿Son públicos las profesiones o cargos públicos de los órganos de gobierno? & 17,05 & 10,28 \\
\hline $\begin{array}{l}\text { b.3. ¿Son públicos la relación de parentesco y afinidad con otros miembros } \\
\text { del órgano de gobierno y con el equipo directivo de la organización? }\end{array}$ & 1,55 & 1,22 \\
\hline b.4. ¿Son públicos los currículos vitae de los miembros del equipo directivo? & 10,98 & 8,54 \\
\hline $\begin{array}{l}\text { b.5. ¿Son públicos la relación entre los miembros del órgano de gobierno } \\
\text { y los proveedores y coorganizadores de la actividad? }\end{array}$ & $12,40 * * *$ & $0,00^{* * * *}$ \\
\hline c. ¿Son públicos en la web la renovación de los miembros del órgano de gobierno? & $17,83 * * *$ & $1,22 * * *$ \\
\hline $\begin{array}{l}\text { d. ¿Son públicos en la web los mecanismos aprobados por el órgano de gobierno } \\
\text { que eviten situaciones de conflicto de interés en el seno del órgano de gobierno? }\end{array}$ & 5,43 & 2,44 \\
\hline $\mathrm{p}<0,001 * * * ; \mathrm{p}<0,05^{* * ;} ; \mathrm{p}<0.1 *$ & & \\
\hline
\end{tabular}

\section{Conclusiones}

La transparencia es una cuestión ineludible para mitigar los prejuicios de malversación de recursos y fortalecer la legitimidad de las ONG como agentes al servicio de la sociedad. Está dirigida para satisfacer las necesidades informativas de los diferentes stakeholders; por tanto, debe ser accesible, de forma que Internet, y en especial las páginas web, es un elemento estratégico para la divulgación de información.

En España, entre las iniciativas puestas en marcha para el fomento de la transparencia del sector, señalamos la labor de la Fundación Lealtad, entidad sin ánimo de lucro que evalúa la transparencia de aquellas ONG interesadas a través del cumplimiento de los nueve principios de transparencia que constituyen su "Guía 
de la Transparencia y las Buenas Prácticas de las ONG”. Dada la acogida de este código de conducta, la Fundación pretende adaptar los principios de su guía a las necesidades informativas de los stakeholders de las ONG de Latinoamérica y, en concreto, de México.

En este trabajo se ha analizado la transparencia on-line de las ONG mexicanas y españolas a través de un índice de visibilidad de la transparencia en la web. En este sentido, es preciso remarcar que el estudio del comportamiento de las ONG españolas se centra en el colectivo de ONG que voluntariamente son analizadas según el cumplimiento de los principios de la Fundación Lealtad $\left(\mathrm{ONG}_{E A F L}\right)$. A pesar de este sesgo, encontramos interesante el hecho de conocer la conducta de las ONG $E A F L$, ya que pueden considerarse como indicadores del posible comportamiento que puedan tomar aquellas ONG mexicanas que decidan aplicar a su organización los principios de la Fundación Lealtad.

Del análisis efectuado se desprende que, en términos generales, las ONG de España $\left(\mathrm{ONG}_{E A F L}\right)$ y de México disponen de escasa información en sus portales web; la más divulgada es aquella que hace referencia directa a los objetivos y valores de la organización. Por tanto, existe una tendencia a desarrollar páginas web con contenidos de carácter descriptivo y que no requiere actualización.

A pesar de estos resultados, se muestra que la transparencia on-line de las ONG $E A F L$ es superior a la alcanzada por las ONG mexicanas. Concretamente, los datos revelan que las $\mathrm{ONG}_{E A F L}$ son más proclives a rendir cuentas sobre las actividades que realizan a lo largo del año y a demostrar el estado financiero de sus cuentas. Esta actitud obedece al interés por recuperar la falta de confianza causada por los casos de malversación de recursos ocasionados en los últimos años.

En relación con las ONG mexicanas, hay que resaltar que más de un cuarto por ciento de estas entidades no presentan página web, por lo que es preocupante que las ONG mexicanas no utilicen, al día de hoy, esta herramienta. Este hecho puede deberse a los limitados recursos económicos que disponen estas organizaciones como consecuencia del contexto económico y político en el que se desenvuelven. Entre la escasa información divulgada cabe señalar que, a pesar de ser las ONG mexicanas uno de los principales grupos de presión en la lucha contra la corrupción del país, muestran un bajo interés en justificar la gestión de los fondos recaudados por la organización. 
Por tanto, como principal contribución de este trabajo es el llamado a autoridades, organismos y a las propias ONG, por realizar mayores esfuerzos para promover el uso de Internet como medio de divulgación de información. Estos resultados son de gran interés, pues las ONG deben valorar más la utilidad de la información disponible en sus páginas webs, ya que manteniendo informados a los diferentes stakeholders con información relevante y de calidad se aumentará la confianza en el sector. En este sentido, creemos conveniente la necesidad de regularizar la rendición de cuentas de estas entidades en México a través de una posible reforma de la Ley Federal de Transparencia y Acceso a la Información Pública Gubernamental del 11 de junio de 2002 de manera que se incluya a este colectivo.

Por otro lado, este análisis comparativo evidencia la influencia positiva de la labor de los organismos que fomentan la transparencia en la divulgación de información en red. Por tanto, consideramos que el proyecto de adaptación de los principios de la Fundación Lealtad a la realidad de las ONG mexicanas es una alternativa para mejorar la transparencia on-line de las ONG autóctonas del país.

\section{Referencias}

Argandoña, A. (2007). Ethical management sistems for not for profit organizations, IESE-Business School University of Navarra.

Berman, H. (2006). Public trust and good governance: An essay, Inquiry - Excellus Health Plan, N. 43, Vol. 1.

Bonbrigth, D. (2007). El rostro cambiante de la rendición de cuentas de las ONG. Seminario Internacional Pregonar con el ejemplo. Sociedad Civil y rendición de cuenta. Montevideo

Burger, R. y T. Owens (2010). Promoting transparency in the NGO sector: examining the availability and reliability of self-reported data. World development, Vol. 38, N. 9, pp. 1263-1277.

Brumley, K.M. (2010). Understanding mexican NGOs: goals, strategies, and the local context. Qualitative Sociology, Vol. 33, No. 3, pp. 389-414. 
Caba, C. y C. Castillo (2002). Empresas cotizantes en el mercado de capitales mexicano ante los nuevos instrumentos de comunicación de la información financiera, Hitos de Ciencias Económico Administrativas, N.22, pp. 103-112.

Campbell, D., G. Moore y P. Shrives (2006). Cross-sectional effects in community disclosure, Accounting, Auditing \& Accountability Journal, Vol. 19, No.1.

Choi, F.D. S. (1973). Financial disclosure and entry to the European capital market, Journal of Accounting Research, Vol. 11, pp. 159-175.

Chow, C.W. y A. Wong-Boren (1987). Voluntary financial disclosure by Mexican corporations, The Accounting Review, Vol. 62, No. 3 .

Dhaliwal, D.S. (1980). Improving the quality of corporate financial disclosure, Accounting and Business Research, Vol.10, pp.385-391.

Duble, A.S (1994). Las fundaciones en Europa. protagonismo, legislación, transparencia y autorregulación, CIRIEC-España, Revista de Economía Pública, Social y Cooperativa, No. 17, pp. 193-208.

Fuentes, J. (B2007). Las organizaciones no lucrativas: necesidades de los usuarios de la información financiera. Revista Española del Tercer Sector, No. 6, pp. 91-118.

Fundación Lealtad Guía de la transparencia y de las buenas prácticas [en línea]. (2010). 6 de junio de 2010 [fecha de consulta: 25 de septiembre de 2010]. Disponible en: http://www.guiatransparenciaong.org/guiaong/guía

Gandía, J.L. (2001). La divulgación de información financiera en la era digital, Asociación Española de Contabilidad y Administración de Empresas, Madrid.

García, J.L. (2004. Las cuentas de la economía social. El tercer sector en España, Madrid, Civitas Ediciones.

Grabulosa, J.L. (2007). El debate sobre la transparencia y la rendición de cuentas [en línea]. 25 de Octubre de 2007 [fecha de consulta: 25 de junio del 2009]. Disponible en: http://www.observatoritercersector.org/pdf/publicacions/articulo_boletin_18.pdf 
Greenlee, J., M. Fischer, T. Gordon y E. Keating (2007). An investigation of fraud in Nonprofit Organizations: Occurrences and deterrents. Nonprofit and Voluntary Sector Quarterly, Vol. 36, No. 4, pp. 676-694.

Guberty (2009). Signaling virtue: voluntary accountability programs among nonprofit organizations. Policy Sciences, Vol. 42, No. 3, pp 243-273.

Hackler, D. y G.D. Saxton (2007). The strategic use of information technology by Nonprofit Organization: increasing capacity and untapped potential. Public Administration Review, N. 67,Vol. 3.

Herranz de la Casa, J.M. (2007). La gestión de la comunicación como elemento generador de transparencia en las organizaciones no lucrativas. CIRIEC-España, Revista de Economía Pública, Social y Cooperativa, N. 57, pp. 5-31.

Herawaty, M. y Z. Hoque (2007). Disclosure in the annual reports of Australian government departments. A research note. Journal of Accounting \& Organizational Change, Vol. 3, No. 2, 2007, pp. 147-168.

Hooper, K., R. Sinclair y D. Hui (2008). Financial reporting by New Zealand charities: finding a way forward. Managerial Auditing Journal, Vol. 23, No. 1, pp.68-83.

Ingenhoff, D. y A. M. Koelling (2007). The potential of web sites as a relationship building tool for charitable fundraising NPOs. Public Relations Review, Vol. 35, No. 1, pp. 66-73.

Kang, S. y H. E. Norton (2004). Nonprofit organizations' use of the World Wide Web: are they sufficiently fulfilling organizational goals? Public Relations Review, No. 30, pp. 279-284.

Larran, M. y B. Giner (2002). The use of the internet for corporate reporting by Spanish companies. The International Journal of Digital Accounting Research, Vol. 2, nº1, pp. 53-82.

Lee, T.E., J.Q Chen y R. Zhang (2001). Utilizing the internet as a competitive tool for Non-profit Organizations. The Journal of Computer Information Systems, Vol. 41, No. 3. 
Lister, L. (2003). NGO legitimacy: technical issue or social construct? Critique of Anthropology,Vol. 23, No. 2, pp.175-192.

MacDonald, C., M. McDonnald y W. Norman (2002). Charitable conflicts of interest. Journal of Business Ethics, No. 39, pp. 67-74.

Marcuello, M., A. Bellostas, J. Camón, C. Marcuello, Ana C. Laliena y E. Ortas (2007): Transparencia y sostenibilidad en las empresas de inserción aragoneses. Fundación Economía Aragonesa, No. 42.

McGann, J. y M. Johnstone (2006). The power shift and the NGO credibility crisis. The International Journal of Not-for-Profit Law, Vol. 8, No.2, pp. 65-77.

Medina, J.M. (2009). Transparencia y buen gobierno en las ONGD. Revista Española del Tercer Sector, pp. 93-113.

Moneva, J.M. y A. Bellostas (2007). Accountability en las entidades sin fines lucrativos Centros de Investigación de Economía y Sociedad.

Naudé, A. M., J. D. Froneman y R. A. Atwood (2004). The use of the internet by ten South African non-governmental organizations a public relations perspective. Public Relations Review, Vol. 30, pp. 87-94.

Neal, R. (2008). The importance of the state: political dimensions of a nonprofit network in Oaxaca, Mexico. Nonprofit and Voluntary Sector Quarterly, Vol. 37, No. 3, pp. 492-511.

O'Rourke, D. (2006). Multi-stakeholder regulation: privatizing or socializing global labor standards? World development, Vol. 34, No. 5, pp. 899-918.

Oyelere, P., F. Laswad y R. Fisher (2003), Determinants of internet financial reporting by New Zealand companies. Journal of International Financial, Management and Accounting, Vol. 14, N. 1, pp. 26-63.

Ozcelik, Y. (2008). Globalization and the internet: digitizing the nonprofit sector. Journal of Global Business Issues, Vol. 2, No. 1. 
Pérez, V. y C. Mateos (2006). ONG, internet y comunicación alternativa en las ONG dedicadas a la inmigración en España. Razón y Palabra, N.29.

Peregrine, M. y J. Schwartz (2004). Using your web site as an accountability tool. Trustee, Vol. 57, No. 5.

Robbins, W.A. y K. R. Austin (1986). Disclosure quality in governmental financial reports: an assessment of the appropriateness of a compound measure. Journal of Accounting Research, Vol. 4, No.2, pp. 412-421.

Rodríguez, G. (2004). Factores explicativos de la revelación voluntaria de información sobre fuentes de ventaja competitiva empresarial. Revista Española de Financiación y Contabilidad, Vol.33, N. 122, pp. 705-739.

Ruíz, J.I. (2001). El sector no lucrativo en España. Ciriec España, Revista española de Economia, Pública y Social, No. 37, pp. 51-58

Ruiz, M. Tirado y P.A.C. Morales (2008). Transparencia y calidad de la información económico-financiera en las entidades no lucrativas. Un estudio empírico a nivel andaluz. CIRIEC-España, Revista de Economía Pública, Social y Cooperativa, No. 63, pp. 253-274.

Serrano, C., M. Rueda y M.P. Portillo (2008). Factors influencing e-disclosure in local public administrations. Documentos de trabajo, No. 3.

Salomon, L. et al. (2001). La sociedad civil global. Las dimensiones del sector no lucrativo, Bilbao. Fundación BBVA

Sanz, C. J. y A. C. Koc (2006). Transparencia de la información en internet del sector no lucrativo en España. Partida Doble, No. 183, pp. 96-105.

Seaman (2006). What works best, The Internal Auditor, Vol. 63, No. 1.

Sorj, B. (2007). ¿Pueden las ONG reemplazar al Estado? Sociedad civil y Estado en América Latina, Nueva Sociedad, No. 20, pp.126-140.

Rathgeb, S. (2008). The challenge of strengthening nonprofits and civil society. Public Administration Review. 
Treviño (2004). Las ONG de derechos humanos y la redención de la soberanía del estado mexicano, Foro Internacional, No. 177, Vol. 3, pp. 509-539.

Vaccaro, A. y P. Madsen (2009). ICT and an NGO: Difficulties in attempting to be extremely transparent, Ethics and Information Technology

Wanderley, L.S.O., R. Lucian, R. Farache y J. Milton (2008). CSR Information disclosure on the web: a context-based approach analysing the influence of country of origin and industry sector, Journal of Business Ethics, No. 82, pp.369-378. 
